\title{
Die Bedeutung der Pharmaindustrie in Deutschland
}

Jasmina Kirchhoff

Inhaltsverzeichnis

$7.1 \quad$ Einleitung -94

7.2 Die Pharmaindustrie am Standort Deutschland - 95

7.2.1 Pharmaunternehmen am Wirtschaftsstandort - 95

7.2.2 Pharmazeutische Forschung in Deutschland - 98

7.3 Die deutsche Pharmaindustrie im europäischen Vergleich - 100

$7.4 \quad$ Fazit -101

Literatur - 102 


\section{- Zusammenfassung}

Eine starke innovative Pharmaindustrie ist wichtig für die Zukunftsfähigkeit des deutschen Wirtschaftsstandorts und ein starker Wirtschaftsstandort Deutschland ist wichtig für eine global wettbewerbsfähige Pharmaindustrie vor Ort. Pharmazeutische Unternehmen sind in Deutschland auf allen Stufen der pharmazeutischen Wertschöpfungskette tätig. Mit ihren wirtschaftlichen Aktivitäten und ihrer Exportstärke schaffen sie hochwertige Arbeitsplätze, erwirtschaften eine überdurchschnittliche Wertschöpfung und leisten mit ihren Innovationen und über ihre Einbindung in nationale und internationale Forschungsund Produktionsnetzwerke einen positiven gesamtwirtschaftlichen Beitrag zu Wohlstand und Beschäftigung. Für eine auch in $\mathrm{Zu}$ kunft wettbewerbsfähige Arzneimittelentwicklung und -produktion in Deutschland braucht es eine klug aufgestellte Industriepolitik. Pharmazeutische Unternehmen müssen sich darauf verlassen können, dass sie ihre Produkte langfristig wirtschaftlich auf dem deutschen und europäischen Markt absetzen können, wenn sie die hohen Investitionen für den Aufbau neuer Forschungs- und Produktionsanlagen vor Ort aufbringen.

\subsection{Einleitung}

In der Diskussion um Strategien zur Sicherung der zukünftigen Finanzierbarkeit der gesetzlichen Krankenversicherung werden oftmals die Preisentwicklungen innovativer Arzneimittel in besonderem Maße kritisch hinterfragt (Glaeske 2020; Schwabe und Ludwig 2020). Allerdings kann die Angemessenheit aktueller und zukünftiger Preise für patentgeschützte Pharmazeutika nicht ausschließlich auf der Grundlage einer Kostenbetrachtung im Gesundheitswesen bewertet werden. Neben der Berücksichtigung des individuellen und gesellschaftlichen Nutzens innovativer Arzneimittel ist auch die Bedeutung der Branche für den Forschungs- und Wirtschaftsstandort zu berücksichtigen.

In der Corona-Krise zeigt sich der Wert neuer Pharmaprodukte und der Innovationskraft hiesiger Pharmaunternehmen besonders deutlich. Zum einen braucht es effektive Impfstoffe gegen Sars-CoV-2 und Arzneimittel zur wirksamen Behandlung von an Covid-19 erkrankten Menschen. Pharmazeutische Unternehmen entwickelten in einer nie dagewesenen Geschwindigkeit mittlerweile vier in Europa zugelassene Impfstoffe. An diesem Erfolg sind Pharmaunternehmen aus Deutschland maßgeblich beteiligt. Zum anderen führten im Jahr 2020 die in nahezu allen Volkswirtschaften steigenden Infektionszahlen und ergriffenen Maßnahmen zur Eindämmung der Pandemie zu einem massiven Einbruch, auch in der deutschen Wirtschaft. Die exportstarke Pharmaindustrie entwickelte sich jedoch stabiler als viele andere industrielle Sektoren (Kirchhoff und Schumacher 2021, 2021a). In der Diskussion um angemessene Arzneimittelpreise ist daher zu berücksichtigen, dass eine starke Pharmaindustrie vor Ort nicht nur für eine gute und sichere Arzneimittelversorgung wichtig ist, sondern diese aufgrund ihrer Innovationsintensität und Krisenresistenz zudem für einen global wettbewerbsfähigen Wirtschaftsstandort relevant ist.

Will Deutschland bei der Arzneimittelentwicklung und -produktion im globalen Wettbewerb weiterhin eine wichtige Rolle spielen, muss sich der Standort im internationalen Vergleich attraktiver aufstellen. Pharmazeutische Unternehmen profitieren in Deutschland nach wie vor von der guten Infrastruktur, hoch qualifizierten Mitarbeitenden und einer starken Forschungslandschaft. Für einen starken pharmazeutischen Produktionsstandort sind aber nicht nur die industriepolitischen Rahmenbedingungen wie eine wettbewerbsfähige Steuerund Abgabenlast, der Zugang zu hochqualifizierten Fachkräften oder verbesserte Investitionsbedingungen in den Blick zu nehmen für den Auf- und Ausbau pharmazeutischer Produktionen in Deutschland brauchen Unternehmen die Sicherheit, vor Ort auch lang- 
fristig kostendeckend produzieren und wirtschaftlich vertreiben zu können (Bundesverband der deutschen Industrie e. V. 2021; Kirchhoff 2021). Die Erosion des deutschen Produktionsstandorts für generische Wirkstoffe verdeutlicht die möglichen Auswirkungen beständiger Regulierungen mit dem Ziel der Preisreduktion (Hosseini und Baur 2020). Daneben ist eine zukunftsfähige starke Pharmaproduktion eng mit dem Erhalt und Ausbau des Forschungsstandorts verknüpft. Denn ohne eigene Entwicklungskapazitäten im pharmazeutischen Bereich verlöre Deutschland zunehmend den Anschluss im globalen Standortwettbewerb. Vor allem aber würde Deutschland den Ruf eines forschungsorientierten Standorts verspielen. Dies könnte langfristig zur Abwanderung betroffener, aber auch nicht direkt betroffener Industriezweige führen.

Dieser Beitrag zeigt in einem ersten Schritt die ökonomische Bedeutung der Pharmaindustrie am Wirtschaftsstandort sowie den Beitrag der Branche für den Forschungsstandort Deutschland auf. In einem zweiten Schritt wird die deutsche Pharmaindustrie im europäischen Vergleich betrachtet.

\subsection{Die Pharmaindustrie am Standort Deutschland}

\subsubsection{Pharmaunternehmen am Wirtschaftsstandort}

Pharmazeutische Unternehmen beschäftigten im Jahr 2020 rund 115.500 Mitarbeitende und erwirtschafteten einen Umsatz von 47 Mrd. $€$. Knapp zwei Drittel des Umsatzes werden im Ausland erwirtschaftet und damit nicht über das deutsche Gesundheitssystem finanziert, sondern über ausländische Kund:innen und Unternehmen. Im selben Jahr wurden am Standort pharmazeutische Erzeugnisse im Wert von 32 Mrd. $€$ produziert. In Deutschland sind dies vor allem hochwertige verbrauchsfertige Medikamente, Impfstoffe so- wie Zubereitungen für die medizinische Diagnostik: $86 \%$ des Produktionswerts entfallen auf diese pharmazeutischen Spezialitäten. $12 \%$ des hiesigen Produktionswerts sind der Herstellung pharmazeutischer Grundstoffe zuzurechnen, also der Herstellung aktiver pharmazeutischer Substanzen, die für die Medikamentenherstellung benötigt werden. Die hier ansässigen Unternehmen sind damit auf $\mathrm{Zu}-$ lieferungen von Startmaterialien, Hilfs- und Grundstoffen aus dem In- und Ausland angewiesen, soweit sie diese im eigenen Unternehmen nicht selbst herstellen (Grömling und Kirchhoff 2020). Die Vorleistungsquote der Pharmaindustrie, und damit der Anteil der Vorleistungen am Produktionswert der Branche, lag im Jahr 2018 nominal bei knapp über $50 \%$ - im Durchschnitt der industriellen Sektoren beträgt die Vorleistungsquote gut zwei Drittel. Der verbleibende Teil des Produktionswerts entspricht der erwirtschafteten Bruttowertschöpfung, die in der Pharmaindustrie folglich eine deutlich höhere Bedeutung hat als im Verarbeitenden Gewerbe insgesamt (Grömling und Kirchhoff 2020). Im Jahr 2018 erwirtschafteten pharmazeutische Unternehmen eine Bruttowertschöpfung von 17,3 Mrd. $€$. Unternehmerische Investitionen sind entscheidend für die zukünftige Entwicklung des Pharmastandorts. Im Jahr 2019 investierten pharmazeutische Unternehmen vor Ort 2,3 Mrd. $€$ in Anlagen, Gebäude und Maschinen und bekennen sich damit nach wie vor zum Wirtschaftsstandort Deutschland (Institut der deutschen Wirtschaft 2020).

Doch die Betrachtung der absoluten Werte ökonomischer Indikatoren greift zu kurz, um die Bedeutung der Branche für den Wirtschaftsstandort abschließend beurteilen zu können: Erstens zeigt sich die Pharmaindustrie bezogen auf ihre Größe im Vergleich zu anderen industriellen Branchen überdurchschnittlich produktiv und investitionsstark. Zweitens entwickelt sich die Pharmaindustrie im Vergleich zum Durchschnitt des Verarbeitenden Gewerbes auch in Krisenzeiten stabil. Drittens wirkt die Krisenresistenz der Branche über ihre vielfältigen Vorleistungsverflechtungen als 
stabilisierender Faktor auf den gesamten Wirtschaftsstandort.

1) Für die Gestaltung eines zukunftsfähigen Wirtschaftsstandorts braucht es vor allem wertschöpfungs- und investitionsstarke Branchen vor Ort. Dies ist umso entscheidender, als dass sich in einer schrumpfenden wie auch alternden Bevölkerung das Arbeitsangebot im Zeitablauf verkleinert. Diese Entwicklung stellt nicht nur den Wirtschaftsstandort an sich, sondern ebenso die Finanzierbarkeit der sozialen Sicherungssysteme vor große Herausforderungen. Um die Auswirkungen des demografischen Wandels zumindest abfedern zu können, braucht es in Zukunft vermehrt wertschöpfungs- und investitionsstarke Branchen vor Ort. So erwirtschaftete die Pharmaindustrie je Beschäftigten im Jahr 2018 über $125.000 €$ Bruttowertschöpfung und belegte damit knapp nach der chemischen Industrie den zweiten Platz der wertschöpfungsstärksten Branchen am Standort (Institut der deutschen Wirtschaft 2020). Ähnlich stark zeigt sich die Pharmaindustrie hinsichtlich ihrer Investitionsintensität: Mit $18.900 €$ je Beschäftigten investierte die Pharmaindustrie im Jahr 2019 nur knapp weniger als der Fahrzeugbau, welcher nicht ganz $19.000 €$ je Beschäftigten am Standort in materielle Sachanlagen aufwendete.

2) Während der industrielle Sektor Deutschlands aufgrund der gesellschaftlichen und ökonomischen Herausforderungen der Corona-Krise deutliche Einbrüche in seiner konjunkturellen Entwicklung verzeichnete, verblieb die Pharmaindustrie nahezu auf ihrem stabilen Wachstumspfad der Vorjahre (Kirchhoff und Schumacher 2021, 2021a). Der Umsatzindex der Pharmaindustrie lag real und arbeitstäglich bereinigt im Jahr 2020 zwar mit einem Minus von 1,1\% knapp unterhalb des Vorjahreswerts und auch die Auftragseingänge lagen 1,5\% niedriger als noch im Jahr 2019, aber die pharmazeutische Produktion verblieb mit einem leichten Rückgang von $0,3 \%$ auf dem Vorjahresniveau. Das gesamte Verarbeitende Gewerbe musste im Jahr 2020 hingegen

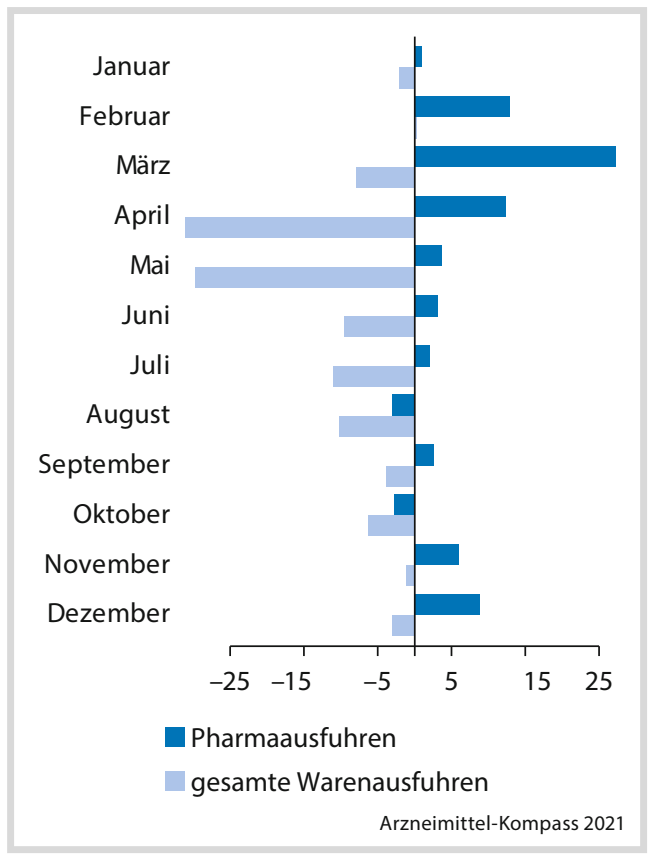

- Abb. 7.1 Veränderung des Ausfuhrwerts im Vorjahresvergleich, 2020, in \% (Quellen: Statistisches Bundesamt, Institut der deutschen Wirtschaft)

Auftragseingangseinbußen von 7,1\% im Vorjahresvergleich hinnehmen; der industrielle Umsatz ging um 10,1\% zurück, die Produktion sank um 10,6\%. Auch die Entwicklung der Ausfuhren pharmazeutischer Erzeugnisse zeigt die Krisenresistenz der Pharmaindustrie im Vergleich zum industriellen Durchschnitt deutlich auf (- Abb. 7.1). Anders als die Exporte insgesamt konnte der Wert der ausgeführten pharmazeutischen Erzeugnisse nahezu über das gesamte Jahr 2020 gesteigert werden.

Alle Länder versuchen sowohl die Produktion als auch die Lieferfähigkeit von Wirkstoffen und Arzneimitteln in der Corona-Pandemie unter den teilweise massiven Einschränkungen durchgängig aufrecht zu erhalten, um die pharmazeutischen Wertschöpfungsketten und damit die Arzneimittelversorgung der Bevölkerung nicht zu gefährden. Von einem grundsätzlich gesteigerten Arzneimittelbedarf aufgrund der epidemiologischen Lage kann dage- 


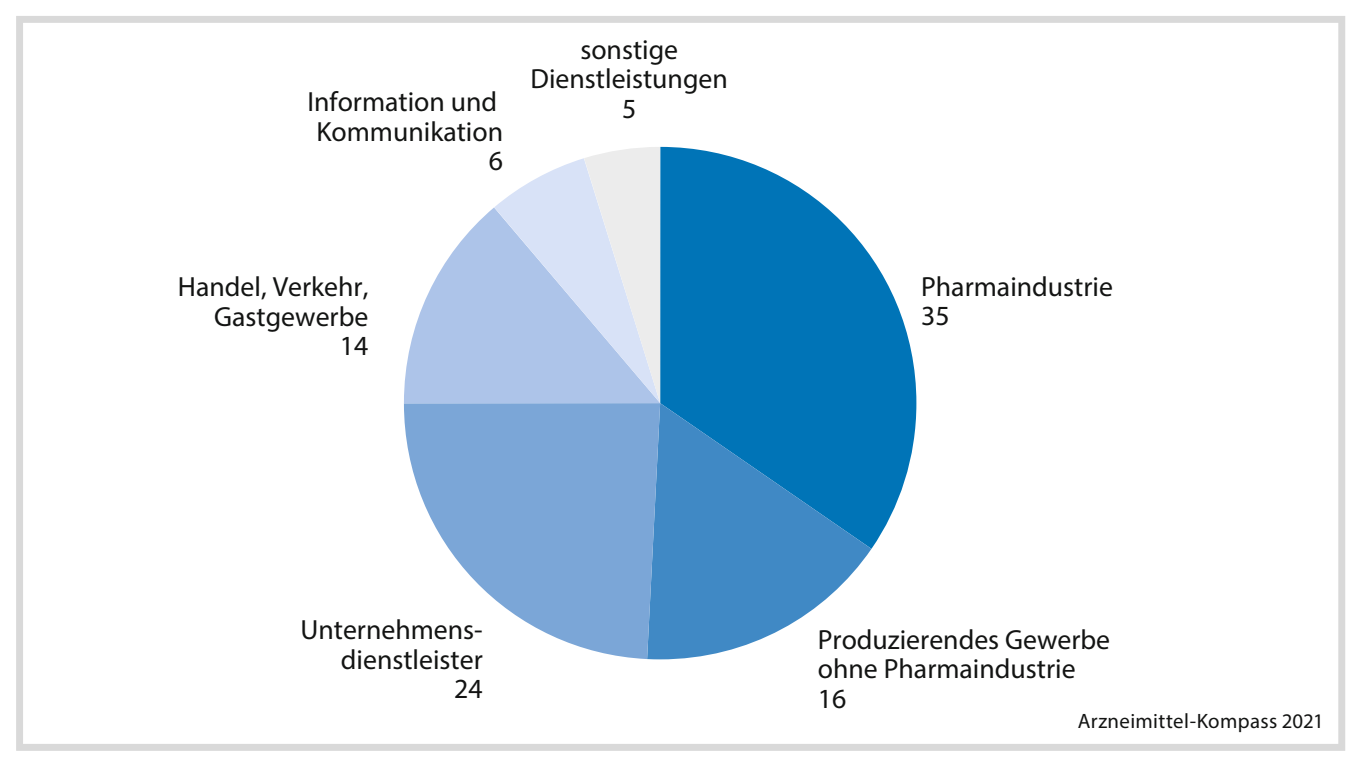

- Abb. 7.2 Inländische Vorleistungslieferanten der Pharmaindustrie, 2017, in \% (Quellen: Statistisches Bundesamt, Institut der deutschen Wirtschaft)

gen nicht ausgegangen werden (Kirchhoff und Schumacher 2021). Doch die robuste Entwicklung der Branche ist nicht allein darauf zurückzuführen, dass Arzneimittel in einer medizinischen Krise unabdingbar sind. So sind pharmazeutische Lieferketten über eine vergleichsweise hohe Vorratshaltung oftmals besser abgesichert als Lieferketten anderer Branchen (Grömling und Kirchhoff 2020). Zudem ist die pharmazeutische Industrie grundsätzlich weniger abhängig von konjunkturellen Entwicklungen, da sich die Nachfrage nach Arzneimitteln vor allem am medizinischen Bedarf und weniger an wirtschaftlichen Schwankungen orientiert. Dies zeigte sich auch in der Wirtschaftskrise 2008/09, in der die Entwicklung relevanter Konjunkturindikatoren in der Pharmaindustrie stabiler verlief als in anderen industriellen Branchen (Kitanovic 2010).

3) Die Produktion eines Arzneimittels erfolgt in einem komplexen Zusammenspiel von Lieferanten, Sublieferanten, Herstellern und behördlicher Aufsicht. Aufgrund dieser Vernetzung mit Akteur:innen aus der eigenen und aus einer Vielzahl anderer Branchen sind pharmazeutische Unternehmen nicht nur Treiber des medizinischen Fortschritts, sondern stabilisieren zudem den gesamten Wirtschaftsstandort. Indem sie Waren und Dienstleistungen von vorgelagerten Branchen beziehen, generieren die beauftragten Lieferanten Umsatz und Wertschöpfung und schaffen Arbeitsplätze. Gut zwei Drittel der von der Pharmaindustrie benötigten Vorleistungen werden dabei von Unternehmen aus dem Inland bezogen (WIOD 2016). Im Jahr 2017 waren ein Drittel ihrer inländischen Vorleistungen dem pharmazeutischen Bereich zuzurechnen, weitere $16 \%$ dem verbleibenden produzierenden Gewerbe; die Hälfte ihrer Vorleistungen stammten von Unternehmen aus dem Dienstleistungsbereich (- Abb. 7.2). Jeder Euro, der in der industriellen Gesundheitswirtschaft erwirtschaftet wird, schafft eine zusätzliche Wertschöpfung von $1,01 €$ in der Gesamtwirtschaft (Bundesministerium für Wirtschaft und Energie 2020) - die pharmazeutische Industrie leistet hierzu einen bedeutenden Beitrag. 


\subsubsection{Pharmazeutische Forschung in Deutschland}

Spitzentechnologiesektoren wie die Pharmaindustrie entwickeln neue, grundlegende Technologien und erweitern so die Wachstumsmöglichkeiten der Wirtschaft (Legler und Frietsch 2006). Für einen global wettbewerbsfähigen Wirtschaftsstandort ist es daher entscheidend, dass Unternehmen der Spitzentechnologie ihre Forschungsstandorte vor Ort belassen, respektive ausbauen.

Über 21.000 Beschäftigte (in Vollzeitäquivalenten) waren im Jahr 2018 in den Forschungsabteilungen pharmazeutischer Unternehmen beschäftigt. Insgesamt wendete die Pharmaindustrie 7,8 Mrd. $€$ für ihre Aktivitäten in der Forschung und Entwicklung (FuE) auf und ist damit nach dem Kraftfahrzeugbau und der Elektronik der drittstärkste forschende Industriezweig des Verarbeitenden Gewerbes (• Abb. 7.3). FuE-Aktivitäten sind jene Tätigkeiten, die die Entstehung und Verwendung neuen Wissens zum Ziel haben
(OECD 2015). In der pharmazeutischen Industrie umfasst dieser Prozess die Erforschung neuer Wirkstoffkandidaten sowie die vorklinischen und klinischen Studien zur Zulassung des neuen Medikaments. Auch nach seiner Zulassung durchläuft das Medikament weitere klinische Studien, um Langzeitdaten zu seiner Wirksamkeit und Verträglichkeit sowie zu Neben- und Wechselwirkungen zu erhalten (Verband forschender Arzneimittelhersteller e. V. 2018). Der überwiegende Teil der Forschungsaufwendungen erfolgt nach der vorklinischen Prüfung; $84 \%$ der FuE-Aufwendungen fallen für Bereiche des Entwicklungsprozesses außerhalb der Präklinik an (Bundesverband der pharmazeutischen Industrie e. V. 2020).

Die Pharmaindustrie gilt als die forschungsintensivste Branche in Deutschland, denn keine andere Branche wendet einen höheren Anteil ihres Umsatzes für Forschungsaktivitäten auf. Über $13 \%$ des Umsatzes der pharmazeutischen Unternehmen flossen im Jahr 2017 in die FuE; darauf folgten die Elektronik mit einer Forschungsintensität von

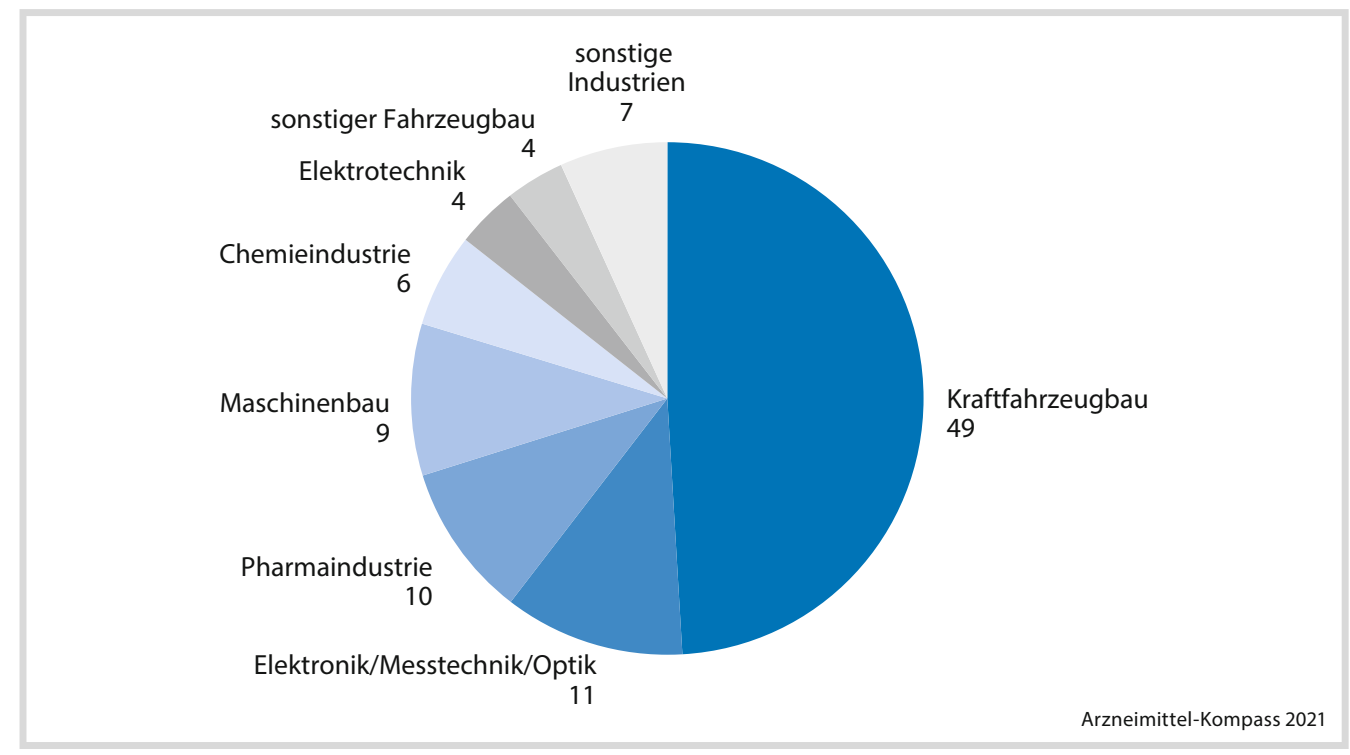

- Abb. 7.3 FuE-Aufwendungen im Verarbeitenden Gewerbe nach Branchen, 2018, in \% (Quellen: Stifterverband für die deutsche Wissenschaft, Institut der deutschen Wirtschaft) 


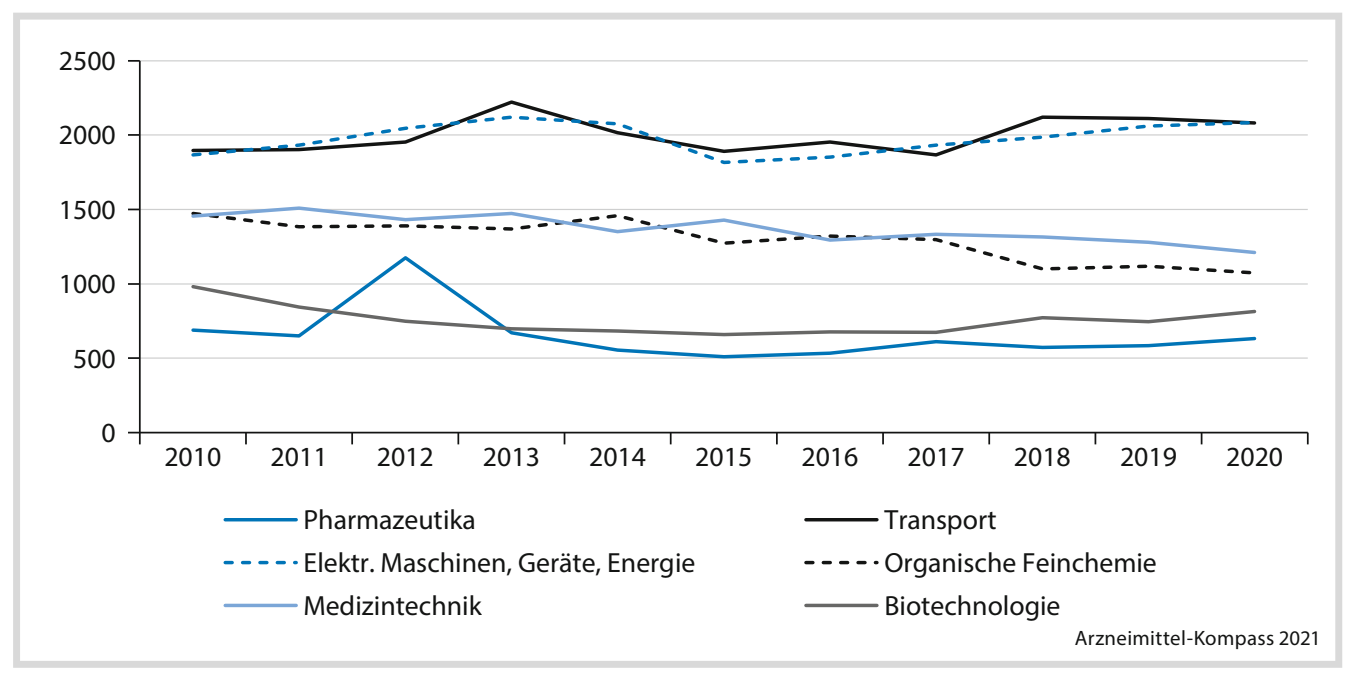

- Abb. 7.4 Anzahl europäischer Patentanmeldungen aus Deutschland in ausgewählten Technologiefeldern (Quellen: Europäisches Patentamt, Institut der deutschen Wirtschaft)

8,5\% und der Kraftfahrzeugbau mit knapp $8 \%$ (Stifterverband 2019).

Mit 5,2 Mrd. $€$ fand der überwiegende Teil der Forschungsaufwendungen pharmazeutischer Unternehmen im Jahr 2018 Verwendung in hausinternen Forschungsaktivitäten. 2,6 Mrd. $€$ wurden für externe FuE aufgewendet; hierbei handelt es sich um Aufwendungen der Unternehmen für FuE-Leistungen, die andere Unternehmen, Universitäten oder Forschungsinstitutionen für sie im Auftrag erbringen (Stifterverband 2021). Die pharmazeutische Forschung setzt seit Jahren zunehmend auf kooperative Innovationsprozesse. Die internen FuE-Aufwendungen stiegen im Zeitraum 2010 bis 2018 um $40 \%$, die externen FuE-Aufwendungen legten um $141 \%$ zu. Die Zunahme strategischer Allianzen in der Pharmaforschung wurde unter anderem von einer steigenden Komplexität der Forschungsprozesse, neuen technologischen Möglichkeiten und einem steigenden Kostendruck der Gesundheitssysteme getrieben (Kirchhoff et al. 2020). Pharmazeutische Unternehmen sind vermehrt in nationale und internationale Forschungsnetzwerke eingebunden, die unternehmerische und akademische Forschung zusam- menbringen. Die Zusammenarbeit mit unterschiedlichen Akteur:innen entlang der gesamten pharmazeutischen Wertschöpfungskette kann zu einer Erhöhung der FuE-Produktivität führen (de Vrueh und Crommelin 2017).

Für Analysen der Innovationskraft von Unternehmen, Branchen und Volkswirtschaften werden maßgeblich Patentdaten herangezogen. Patente gewährleisten den Anmeldenden für einen Zeitraum von in der Regel 20 Jahren das ausschließliche Nutzungsrecht an der Erfindung. Im Gegenzug veröffentlichen die Anmeldenden die technischen Details der Erfindung. Deutsche Unternehmen meldeten beim Europäischen Patentamt im Jahr 2020631 Patente im Technologiefeld der Pharmazeutika an - der höchste Wert seit 2013. Zwar entwickelten sich die pharmazeutischen $\mathrm{Pa}-$ tentanmeldungen in den letzten Jahren stabil, doch liegt die Zahl in anderen Technologiefeldern zum Teil deutlich höher (• Abb. 7.4). So sind im pharmazeutischen Forschungsprozess nahezu ausschließlich Unternehmen aus der Pharmaindustrie aktiv; 93\% aller internen FuE-Aufwendungen im Erzeugnisbereich Pharmazie kommen aus der eigenen Branche; im Erzeugnisbereich der Elektronik und Elek- 
trotechnik liegt dieser Anteil bei 63\% (Stifterverband 2019). Vor allem aber ist die Pharmaforschung sehr risikobehaftet und kostenintensiv. Im Durchschnitt dauert es 13 Jahre und kostet 1 bis 1,6 Mrd. US-Dollar, bis ein Medikament auf den Markt kommen kann (Verband forschender Arzneimittelhersteller e. V. 2015). Von 5.000 bis 10.000 Substanzen, die während der Forschungsphase neu synthetisiert werden, schaffen es im Durchschnitt zwölf Substanzen in die Phase der vorklinischen Prüfung, bei der in Laborversuchen insbesondere die Toxizität des Wirkstoffkandidaten getestet wird; auf dieser Stufe erfolgt in der Regel die Anmeldung zum Patent (Graetsch 2020). Von diesen Kandidaten gehen neun in die Erprobung am Menschen, ein Kandidat erreicht die Zulassung (Verband forschender Arzneimittelhersteller e. V. 2018). Pharmazeutische Unternehmen nehmen das sehr hohe unternehmerische Risiko einer Arzneimittelentwicklung auf sich, wenn ihnen die Rekapitalisierung der hohen Forschungsaufwendungen bei Markteintritt möglich erscheint - aufgrund der frühen Patentanmeldung steht ein Arzneimittel nach erfolgreicher Zulassung in der Regel noch zwölf Jahre unter Patentschutz (Verband forschender Arzneimittelhersteller e. V. 2018a).

\subsection{Die deutsche Pharmaindustrie im europäischen Vergleich}

Im Jahr 2019 wurden knapp 49\% des Umsatzes im Weltpharmamarkt in Nordamerika generiert, $23 \%$ in Europa (European Federation of Pharmaceutical Industries and Associations 2020). Zwar holten einige Schwellenländer in der Vergangenheit auf und wurden für pharmazeutische Unternehmen zu immer wichtigeren Absatz- und Produktionsstandorten (Kirchhoff 2017). Jedoch wird die Liste der zehn größten Einzelmärkte der Pharmaindustrie nach wie vor von Ländern Nordamerikas und Europas dominiert, lediglich China und Brasilien als Länder außerhalb dieser beiden Wirtschaftsräume belegen Platz 2 respekti- ve Platz 9. Deutschland positionierte sich im Jahr 2019 mit einem Anteil von 4,2\% auf dem vierten Rang der wichtigsten Einzelmärkte am Weltpharmamarkt - hinter den USA, China und Japan (Bundesverband der pharmazeutischen Industrie e. V. 2020). Im Vergleich zum Jahr 2010 konnte Deutschland zwar seine Position halten, doch die USA bauten ihren Vorsprung aus, während China deutlich zulegte und an Japan vorbeizog. Der Gesamtumsatz auf dem Weltpharmamarkt stieg im Zeitraum 2010 bis 2019 jahresdurchschnittlich um 5,3\%. Die Länder der Europäischen Union konnten ihren gemeinsamen Umsatz im selben Zeitraum um jahresdurchschnittlich 3,1 \% steigern, Deutschland legte jedes Jahr um durchschnittlich 4,1\% und China sogar um 12,3\% p. a. zu, die USA steigerten sich um 7,9\% p. a. (Bundesverband der pharmazeutischen Industrie e. V. 2020, 2011).

Europa als einer der größten Pharmamärkte weltweit ist nach wie vor ein traditionell wichtiger Standort für die wirtschaftlichen Aktivitäten pharmazeutischer Unternehmen. Dabei zeigt sich Europa hinsichtlich der wirtschaftlichen Stärke und Entwicklung seiner nationalen Pharmastandorte heterogen. Neben der Schweiz gelten Deutschland, Frankreich, Italien, das Vereinigte Königreich und Spanien als die größten Produktionsstandorte Europas (im folgenden EU-27, inklusive der Schweiz und dem Vereinigten Königreich). $54 \%$ der Bruttowertschöpfung der in Europa ansässigen Pharmaindustrie wurden im Jahr 2018 in diesen sechs Ländern erwirtschaftet, zwei Drittel der Pharmabeschäftigten Europas arbeiteten in diesen Ländern. Deutschland sticht nicht nur als wichtigster europäischer Absatzmarkt hervor. Im Jahr 2018 trug die deutsche Pharmaindustrie $15 \%$ des Produktionswerts und $14 \%$ der Bruttowertschöpfung (• Abb. 7.5) - lediglich die Schweiz zeigte sich in diesen beiden ökonomischen Kenngrößen stärker. Mit einem Anteil von $22 \%$ arbeiteten die meisten Pharmabeschäftigten Europas in Deutschland.

Die deutsche Pharmaindustrie ist im Bereich der FuE und Innovation im europäischen Vergleich ebenfalls stark aufgestellt. $25 \%$ der 


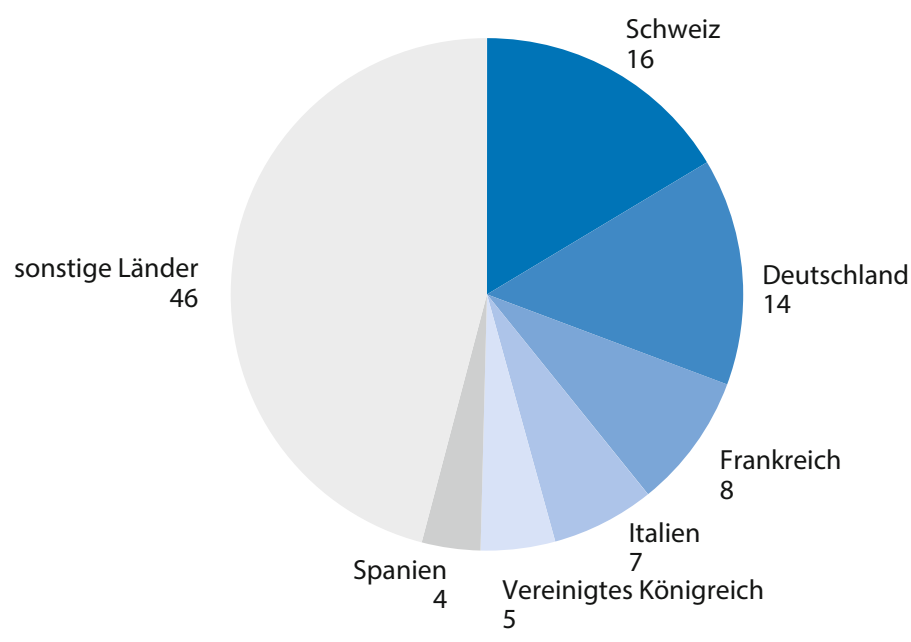

Arzneimittel-Kompass 2021

- Abb. 7.5 Bruttowertschöpfung der Pharmaindustrie in Europa (EU-27, inklusive Schweiz und Vereinigtem Königreich) nach Ländern, 2018, in \% (Quellen: Eurostat, Institut der deutschen Wirtschaft)

internen FuE-Aufwendungen, die die Pharmaindustrie in Europa im Jahr 2017 aufwendete, entfielen auf in Deutschland ansässige Unternehmen. Deutschland liegt damit knapp hinter der Schweiz auf dem zweiten Rang (Kirchhoff und Schumacher 2021b). Im Jahr 2020 wurden von europäischen Unternehmen 3.472 pharmazeutische Patente beim Europäischen Patentamt angemeldet (Europäisches Patentamt 2021). Mit einem Anteil von $18 \%$ entfallen die meisten dieser Patente auf deutsche Unternehmen, knapp gefolgt von Frankreich (17\%) und der Schweiz (14\%).

Die deutsche Pharmaindustrie ist nicht nur ein bedeutender Treiber für den heimischen Wirtschafts- und Forschungsstandort und steht dabei im Vergleich zu den anderen europäischen Pharmastandorten sehr gut da, sondern die hiesigen Unternehmen sind zudem eng in den europäischen Pharmastandort eingebunden und stärken diesen über ihre vielfältigen Vorleistungsverflechtungen: $72 \%$ der von pharmazeutischen Pharmaunternehmen importierten Vorleistungen kommen aus der EU, $73 \%$ der von der deutschen Pharmaindustrie exportierten Vorleistungen verblei- ben in dieser Ländergruppe (WIOD 2016). Gleichwohl zeigt der internationale Vergleich der Pharmastandorte, dass sich andere Länder wie die USA oder China in der Vergangenheit besser entwickelt haben und Deutschland Gefahr läuft, seinen Rang zu verlieren. Die Gründe für diese Entwicklung sind aufgrund der Komplexität der Standortentscheidungen global aufgestellter Pharmaunternehmen vielfältig und umfassen beispielsweise einen im internationalen Vergleich zu hohen regulatorischen Aufwand, eine überdurchschnittliche Steuerbelastung der Unternehmen aber auch eine geringere Gründungs- und Risikobereitschaft (Bundesverband der deutschen Industrie e. V. 2021; Kirchhoff 2021).

\subsection{Fazit}

Der Forschungs- und Wirtschaftsstandort Deutschland ist wichtig für die pharmazeutische Industrie - und gleichzeitig ist eine starke Pharmaindustrie vor Ort wichtig für einen global wettbewerbsfähigen Forschungs- 
und Wirtschaftsstandort. Pharmazeutische Unternehmen sind in Deutschland auf allen Stufen der pharmazeutischen Wertschöpfungskette tätig - sie forschen, entwickeln und produzieren hochwertige Arzneimittel und Diagnostika vor Ort und vertreiben ihre Produkte von Deutschland aus im In- und Ausland. Mit ihren wirtschaftlichen Aktivitäten und ihrer Exportstärke schaffen sie Arbeitsplätze, erwirtschaften eine überdurchschnittliche Wertschöpfung und leisten mit ihren Innovationen und über ihre starken Vorleistungsverflechtungen einen positiven gesamtwirtschaftlichen Beitrag zu Wohlstand und Beschäftigung.

Deutschland ist im globalen Wettbewerb nach wie vor ein wichtiger Standort für die Arzneimittelforschung und -produktion. Doch bereits seit einigen Jahren holen die USA und Länder aus dem asiatischen Raum auf. Investitionen in neue Therapiegebiete wie die Genund Zelltherapie finden mittlerweile überwiegend in diesen Ländern statt. So stieg die Zahl der Unternehmen, die in der regenerativen Medizin tätig sind, in Nordamerika im Zeitraum 2017 bis 2020 um $18 \%$ auf 543, in Asien sogar um knapp $51 \%$ auf 184 - in Europa (inklusive Israel) waren im Jahr 2020238 Unternehmen in der regenerativen Medizin tätig und damit $2 \%$ mehr als noch im Jahr 2017 (Alliance for Regenerative Medicine 2020, 2018). Neben der schleichenden Verlagerung der pharmazeutischen Forschung und Produktion zeichnete sich in den letzten Jahren in einigen Ländern ein zunehmender Protektionismus ab, wie in den USA, aber auch in der EU; dies ist gerade für eine exportstarke Branche wie der Pharmaindustrie eine bedenkliche Entwicklung. Um in Zukunft als wichtiger Pharmastandort im globalen Wettbewerb bestehen zu können, braucht es eine klug aufgestellte Industriepolitik. Neben der Sicherstellung des freien Warenverkehrs sind verlässliche investitionsund innovationsfreundliche Rahmenbedingungen am Standort unerlässlich. Dies umfasst Maßnahmen wie beispielsweise die Verbesserung der Finanzierungs- und Investitionsbedingungen, ebenso wie die Vereinfachung und Be- schleunigung behördlicher Genehmigungsverfahren für Forschungsprojekte, aber auch für den Bau und die Qualifizierung von Anlagen sowie für die Validierung von Herstell- und Reinigungsprozessen - diese sind in Deutschland im Vergleich $\mathrm{zu}$ anderen Ländern $\mathrm{zu}$ langsam und zu kompliziert aufgestellt. Daneben ist ein wirtschaftlich attraktiver Zugang zum deutschen und europäischen Absatzmarkt ein ebenso wichtiger Faktor für unternehmerische Standortentscheidungen. Nur wenn sich pharmazeutische Unternehmen darauf verlassen können, dass sie ihre Produkte langfristig wirtschaftlich auf dem deutschen und europäischen Markt absetzen können, werden sie die benötigten hohen Investitionen aufbringen, um Forschungs- und Produktionsanlagen vor Ort auszuweiten respektive neu aufzubauen.

\section{Literatur}

Alliance for Regenerative Medicine (2018) 2018 cell and gene therapy - state of the industry. https://alliancerm. org/wp-content/uploads/2018/05/ARM_SOTI_2018_ FINAL.pdf. Zugegriffen: 10. Juni 2021

Alliance for Regenerative Medicine (2020) Resilience in the face of Covid-19. AusBiotech + Invest 2020. https://alliancerm.org/wp-content/uploads/2020/10/ Ausbiotech-State-of-the-Industry-v2.pdf. Zugegriffen: 10. Juni 2021

Bundesministerium für Wirtschaft und Energie (2020) Gesundheitswirtschaft: Fakten und Zahlen, Ausgabe 2019. Ergebnisse der Gesundheitswirtschaftlichen Gesamtrechnung. Bundesministerium für Wirtschaft und Energie, Berlin

Bundesverband der deutschen Industrie e. V. (2021) Strategie für die industrielle Gesundheitswirtschaft: Anker in Krisenzeiten und Wachstumstreiber der $\mathrm{Zu}$ kunft. Bundesverband der deutschen Industrie e. V., Berlin

Bundesverband der pharmazeutischen Industrie e.V. (2011) Pharma-Daten 2011. Bundesverband der deutschen Industrie e. V., Berlin

Bundesverband der pharmazeutischen Industrie e. V. (2020) Pharma-Daten 2020. Bundesverband der deutschen Industrie e. V., Berlin

Europäisches Patentamt (2021) European patent applications per field of technology and per country of residence of the applicant for each individual year from 2011 until 2020. Patent Statistics. https://www.epo.org/about-us/annual-reports- 
statistics/statistics_de.html. Zugegriffen: 16. Apr. 2021

European Federation of Pharmaceutical Industries and Associations (2020) The Pharmaceutical Industry in Figures - Key Data 2020. European Federation of Pharmaceutical Industries and Associations, Brüssel

Glaeske G (Hrsg) (2020) Innovationsreport 2020: Auswertungsergebnisse von Routinedaten der Techniker Krankenkasse aus den Jahren 2017 bis 2018. Universität Bremen, Socium, Bremen

Graetsch D (2020) Ergänzende Schutzzertifikate für Arzneimittel - Ökonomisch-rechtliche Analyse unter besonderer Betrachtung von Wirkstoffkombinationen. Nomos, Baden-Baden

Grömling M, Kirchhoff J (2020) Produktions- und Zulieferstrukturen der deutschen Pharmaindustrie. IWTrends 47(4):23-44

Hosseini M, Baur M (2020) Marktversagen bei der Arzneimittelversorgung am Beispiel von Antibiotika: Covid19 wirft Schlaglicht auf das Problem - ist aber nicht dessen Ursache. ifo-Schnelldienst 73(5):26-29

Institut der deutschen Wirtschaft (2020) Die pharmazeutische Industrie in Deutschland: Ein Branchenportrait. Institut der deutschen Wirtschaft, Köln

Kirchhoff J (2017) Exportschlager Gesundheit: Wachstumstreiber Schwellenländer? IW-Report (6). Köln

Kirchhoff J (2021) Pharmastandort stärken, Vernetzung fördern - Industriepolitische Handlungsempfehlungen für eine zukunftsfähige Pharmaindustrie. IW Policy Paper. Köln (in press)

Kirchhoff J, Mertens A, Scheufen M (2020) Der CoronaInnovationswettlauf in der Wissenschaft: Eine Analyse der wissenschaftlichen Publikationen zur Bekämpfung der Corona-Pandemie und die Bedeutung für den Pharma-Standort Deutschland. IW-Report (17). Köln

Kirchhoff J, Schumacher S (2021) Pharmaindustrie unter Pandemie-Bedingungen stabil: Konjunkturelle Entwicklung der pharmazeutischen Industrie im Jahr 2020. IW-Report (3). Köln

Kirchhoff J, Schumacher S (2021a) Pharmaindustrie stark zum Jahresende. IW-Kurzbericht (16). Köln

Kirchhoff J, Schumacher S (2021b) Daten und Fakten der deutschen Pharmaindustrie. Verband forschender Arzneimittelhersteller e. V., Köln (Gutachten für den Verband forschender Arzneimittelhersteller e. V.)
Kitanovic J (2010) Die forschenden Pharma-Unternehmen im Konjunkturverlauf. IW-Trends 37(1):2-17

Legler H, Frietsch R (2006) Neuabgrenzung der Wissenswirtschaft - forschungsintensive Industrien und wissensintensive Dienstleistungen (NIW/ISI-Listen 2006). Studien zum deutschen Innovationssystem, 222007. Niedersächsisches Institut für Wirtschaftsforschung, Hannover

OECD (2015) Frascati manual 2015 - guidelines for collecting and reporting data on research and experimental development, the measurement of scientific, technological and innovation activities. OECD, Paris

Schwabe U, Ludwig W (Hrsg) (2020) ArzneiverordnungsReport 2020. Springer, Berlin

Stifterverband für die deutsche Wissenschaft (2019) arendi: Zahlenwerk 2019 - Forschung und Entwicklung in der Wirtschaft 2017. Stifterverband für die deutsche Wissenschaft, Essen

Stifterverband für die deutsche Wissenschaft (2021) Forschung und Entwicklung in der Wirtschaft 2019. facts - Zahlen und Fakten aus der Wissenschaftsstatistik. Stifterverband für die deutsche Wissenschaft, Essen

Verband forschender Arzneimittelhersteller e. V. (2015) Statistics 2015: Die Arzneimittelindustrie in Deutschland. Verband forschender Arzneimittelhersteller e. V., Berlin

Verband forschender Arzneimittelhersteller e. V. (2018) So entsteht ein neues Medikament: In Labors und Kliniken - Wie ein neues Medikament entsteht. https://www.vfa.de/de/arzneimittel-forschung/ so-funktioniert-pharmaforschung/so-entsteht-einmedikament.html. Zugegriffen: 29. März 2021

Verband forschender Arzneimittelhersteller e. V. (2018a) So entsteht ein neues Medikament: Innovationen brauchen einen soliden Rahmen. https://www. vfa.de/de/arzneimittel-forschung/so-funktioniertpharmaforschung/so-entsteht-ein-medikament. html/_2-innovationen-brauchen-einen-solidenrahmen. Zugegriffen: 9. Juni 2021

de Vrueh R, Crommelin D (2017) Reflections on the future of pharmaceutical public-private partnerships: from input to impact. Pharm Res 34(5):985-1999

WIOD - World Input-Output Database (2016) World Input-Output Tables, 2016 Release. http://www.wiod. org/database/wiots16. Zugegriffen: 22. März 2021 
Open Access Dieses Kapitel wird unter der Creative Commons Namensnennung 4.0 International Lizenz (http:// creativecommons.org/licenses/by/4.0/deed.de) veröffentlicht, welche die Nutzung, Vervielfältigung, Bearbeitung, Verbreitung und Wiedergabe in jeglichem Medium und Format erlaubt, sofern Sie den/die ursprünglichen Autor(en) und die Quelle ordnungsgemäß nennen, einen Link zur Creative Commons Lizenz beifügen und angeben, ob Änderungen vorgenommen wurden.

Die in diesem Kapitel enthaltenen Bilder und sonstiges Drittmaterial unterliegen ebenfalls der genannten Creative Commons Lizenz, sofern sich aus der Abbildungslegende nichts anderes ergibt. Sofern das betreffende Material nicht unter der genannten Creative Commons Lizenz steht und die betreffende Handlung nicht nach gesetzlichen Vorschriften erlaubt ist, ist für die oben aufgeführten Weiterverwendungen des Materials die Einwilligung des jeweiligen Rechteinhabers einzuholen. 